Table 1. Main general features at baseline.

\begin{tabular}{llll}
\hline & UIP $(\mathbf{n}=\mathbf{1 0 6})$ & NSIP $(\mathbf{n}=\mathbf{8 4})$ & p value \\
\hline Age, years mean \pm SD & $66 \pm 10$ & $63 \pm 10$ & $\mathbf{0 . 0 4 9}$ \\
Women, $\mathrm{n}(\%)$ & $59(56)$ & $49(58)$ & 0.71 \\
Smoker ever, $\mathrm{n}(\%)$ & $51(48)$ & $45(54)$ & 0.46 \\
ILD duration up toABA, months, median [IQR] & $16[4-50]$ & $11[2-36]$ & 0.57 \\
RF & $100(94)$ & $71(85)$ & $\mathbf{0 . 0 4 1}$ \\
ACPA, $\mathrm{n}(\%)$ & $96(91)$ & $75(89)$ & 0.83 \\
FVC (\% of the predicted), mean \pm SD & $82 \pm 21$ & $89 \pm 19$ & $\mathbf{0 . 0 2 5}$ \\
DLCO (\% of the predicted), mean \pm SD & $63 \pm 19$ & $65 \pm 16$ & 0.46 \\
ABA monotherapy, $\mathrm{n}(\%)$ & $45(42)$ & $41(49)$ & 0.38 \\
ABA combined+ MTX // +other cDMARD, $\mathrm{n}(\%)$ & $15(14) / / 46(43)$ & $16(19) / / 27(32)$ & 0.17 \\
Prednisone at baseline, mg/day, median [IQR] & $7.5[5-10]$ & $10[5-10]$ & 0.20 \\
Previous immunosuppressive therapy, $n(\%)$ & & & \\
MTX & $81(76)$ & $68(81)$ & 0.45 \\
Leflunomide & $48(45)$ & $31(37)$ & 0.25 \\
Sulfasalazine & $16(15)$ & $5(6)$ & $\mathbf{0 . 0 4 6}$ \\
Hydroxychloroquine & $24(23)$ & $16(19)$ & 0.55 \\
Anti-TNF drugs & $37(35)$ & $30(36)$ & 0.80 \\
Rituximab & $19(18)$ & $15(18)$ & 0.99 \\
Tocilizumab & $12(11)$ & $12(14)$ & 0.54 \\
\end{tabular}

ABA, abatacept; ACPA, anti-citrullinated protein antibodies; DMARD, disease-modifying antirheumatic drug, ILD, Interstitial lung disease; MTX, methotrexate; NSIP, non-specific interstitial pneumonia; RA, rheumatoid arthritis; TNF, tumor necrosis factor; UIP, usual interstitial pneumonia.

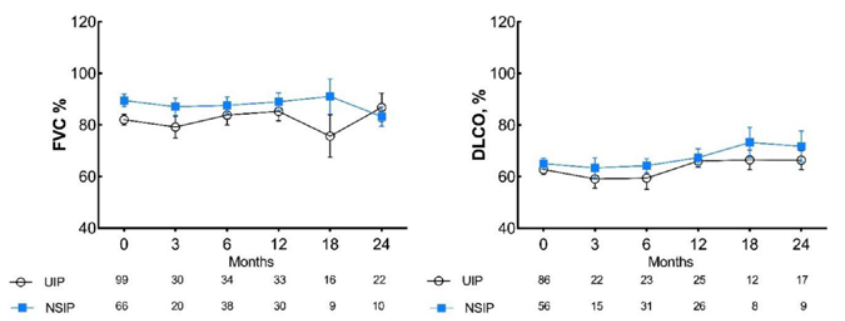

Figure 1. Evolution of pulmonary function tests in RA-ILD patients with UIP and NSIP patterns. FVC and DLCO are expressed as mean $(95 \% \mathrm{Cl})$ and compared between the 2 groups.

Acknowledgements: the Spanish Collaborative Group of Abatacept in Interstitial Lung Disease Associated with Rheumatoid Arthritis

Disclosure of Interests: Belén Atienza-Mateo: None declared, Carlos Fernández-Díaz Speakers bureau: Brystol Myers Squibb, Santos Castañeda: None declared, Rafael Melero: None declared, FRANCISCO ORTIZ SANJUAN: None declared, Ivette Casafont-Solé: None declared, Sebastián C Rodriguez-García: None declared, Iván Ferraz-Amaro: None declared, Miguel A González-Gay: None declared, Ricardo Blanco Speakers bureau: Brystol Myers Squibb DOI: 10.1136/annrheumdis-2021-eular.711

\section{POS0596 THE THERAPEUTIC TARGET IS REACHED MORE OFTEN IN RA PATIENTS STARTING TNF INHIBITORS WITH MODERATE ACTIVITY THAN WITH HIGH DISEASE ACTIVITY - AN ANALYSIS FROM THE ATTRA REGISTRY}

K. Pavelka ${ }^{1}$, L. Nekvindova ${ }^{2}$, J. Zavada ${ }^{1} .{ }^{1}$ Institute of Rheumatology, Rheumatology, Praha 2, Czech Republic; ${ }^{2}$ Institute of Biostatistics and Analyses LTD, Biotatistics, Brno, Czech Republic

Background: Treat to target guidelines recommend achieving remission or low disease activity in rheumatoid arthritis (RA). In the Czech Republic, anti-TNF therapy has been reimbursed only for patients with highly active RA until 2019, when pts with LDA have been also included in the reimbursement policy.

Objectives: The aim of the study was to compare the results of treatment in patients with rheumatoid arthritis and moderate disease activity (MDA) or high disease activity (HDA) starting the therapy with the first anti-TNF drug in routine clinical practice.

Methods: This was a retrospective cohort study of patients treated with the first anti-TNF drug in the Czech Republic. Propensity score was used to match pts starting anti-TNF in MDA or HDA for other important predictive factors. The propensity score was obtained by logistic regression with 10 covariates (gender, age, disease duration, seropositivity, comedication with MTX, comedication with other csDMARDs, number of previosu csDMARDs, anti-TNF drug),. Within the paired groups, one-dimensional logistic models were then calculated to estimate the odds of remission / low activity after 6 and 12 months of treatment.

Results: A total of 2416 patients were analyzed in the study. 2231 patients had high activity (DAS $28>5.1$ ) and 185 patients had moderate activity (3.2<DAS28 $\leq 5.1$ ) at baseline. After 12 months, the low activity state (DAS28 < 3.2) was achieved by patients with MDA in $74.0 \%$ and patients with HDA in $52.2 \%$, (p $<0.001$ ) (FIG 1). The differences btw groups were significant in all evaluated intervals of $0,3,6,12$ months. Furthermore, a comparison of patients with MDA and HAD by propensity score matching, who no longer differ in the baseline characteristics (except for disease activity and quality of life) was performed. Patients with MDA were $2.4 x$ more likely to achieve remission or low activity according to DAS28-ESR after 6 months and 1.7x more likely to achieve remission or low activity after 12 months of treatment compared to patients with HDA at the beginning of treatment.

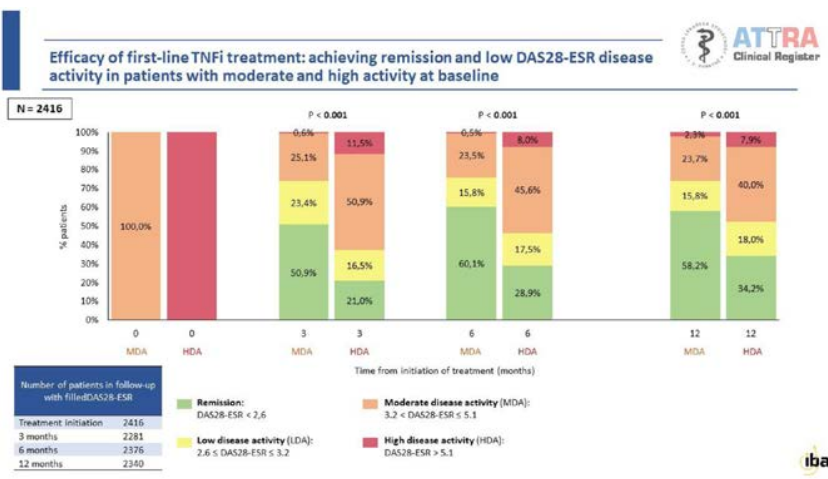

Conclusion: This analysis of the results of treatment with anti-TNF drugs in the ATTRA Registry has shown that starting anti-TNF therapy in patients with RA and MDA leads more often to the target of remission or low activity, than in pts with HDA.

Acknowledgement: Supported by the project of the Ministry of Health Care of the Czech Republic of the conceptual development of the research organization 00023728 Institute of Rheumatology

Disclosure of Interests: Karel Pavelka Speakers bureau: Abbvie, Pfizer, ElliLilly, BMS, UCB, Sanofi, Novartis, Gilead, Paid instructor for: Abbvie, Consultant of: Abbvie, Pfizer, Elli-Lilly, BMS, UCB, Sanofi, Novartis, Gilead, Lucie Nekvindova: None declared, Jakub Zavada Speakers bureau: Abbvie, Elli-Lilly, UCB, Sanofi, Consultant of: Abbvie, UCB, Sanofi, Gilead

DOI: 10.1136/annrheumdis-2021-eular.720

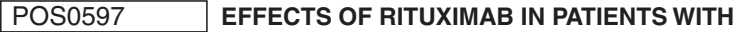 RHEUMATOID ARTHRITIS-RELATED INTERSTITIAL LUNG DISEASE: A SINGLE-CENTRE EXPERIENCE FROM TURKEY}

D. Sahin Eroglu ${ }^{1}$, A. Colaklar ${ }^{2}$, S. Baysal ${ }^{3}$, M. Torgutalp ${ }^{1}$, A. Baygul ${ }^{4}$, M. E. Yayla ${ }^{1}$, T. M. Turgay ${ }^{1}$, G. Kınıkıı ${ }^{1}$, A. Ates ${ }^{1} .{ }^{1}$ Ankara University Faculty of Medicine, Department of Internal Medicine, Division of Rheumatology, Ankara, Turkey; ${ }^{2}$ Ankara University Faculty of Medicine, Department of Radiology, Ankara, Turkey; ${ }^{3}$ Ankara University Faculty of Medicine, Department of Internal Medicine, Ankara, Turkey; ${ }^{4}$ Ankara University Faculty of Medicine, Department of Pulmonology, Ankara, Turkey

Background: Rheumatoid arthritis-related interstitial lung disease (RA-ILD) is the most common type of lung involvement in rheumatoid arthritis (RA). The existence of RA-ILD is associated with worse survival. There is no mainstay treatment for RA-ILD. However, in recent studies, rituximab (RTX) seems to be effective in RA-ILD.

Objectives: To determine the effects of RTX in patients with RA-ILD from a single-centre.

Methods: In our biological treatment outpatient clinic, a retrospective study was conducted in patients with RA who were treated with RTX. Among them, patients with RA-ILD were analysed. For lung response to RTX, progression was defined as a decline of $10 \% \geq$ in forced vital capacity (FVC) and/or a decline of $15 \% \geq$ in diffusion capacity of carbon monoxide (DLCO). Computed tomography of the chest (chest-CT) were integrated to lung response so as to constitute missing data of pulmonary function tests (PFTs).

Results: A total of 165 patients who are followed-up in our biological treatment outpatient clinic have been using RTX for their RA diagnosis. Among 165 patients, 26 (15.8\%) patients with RA-ILD were initiated RTX. Five patients were diagnosed with RA-ILD while using RTX (incidence rate $3 \%$ ). Patients' characteristics were demonstrated in table 1 . Of 26 patients, the most commonly used concomitant disease-modifying antirheumatic drugs was leflunomide (46.2\%) followed by mycophenolate mofetil (11.5), methotrexate (11.5\%) and azathioprine (3.8\%). Twenty-four (92.3\%) patients used steroids. For the evaluation of lung response, 20 patients who had follow-up PFTs and/or chest-CT were compared. 
Median DLCO values for pre- and post RTX were 71.0\% (60.0-77.0) and 63.0\% (47.0-74.0), respectively $(p=0.061)$. Median pre and post-RTX FVC were $74.0 \%$ $(61.0-99.0)$ and $84.0 \%(63.0-100.0)$, respectively $(p=0.28)$. After a median of 2.7 years, $11(55.0 \%)$ patients had stable disease, $2(10.0 \%)$ patients had regression of RA-ILD and 7 (35.0\%) patients had progressive disease. Sex, age, seropositivity and radiographic patterns were not associated with progression. In total, 23 adverse events were detected in $11(42.3 \%)$ patients. Five of them were serious infections requiring hospitalization, mostly pneumonia. Other adverse events were simple infections and surgeries such as arthroplasty. RTX was stopped in $4(15.4 \%)$ patients due to infections secondary to hypogammaglobulinemia in 2 , malignancy in 1 and allergic reaction in 1.

Conclusion: RTX seems to achieve stabilization/improvement of RA-ILD with fewer adverse events. However, given that RA-ILD develops post-RTX, it is not a panacea; therefore, we need more effective treatment modalities in the case of RA-ILD.

Table 1. Characteristics of 26 patients with RA-ILD treated with rituximab

\begin{tabular}{ll}
\hline Age at first RTX infusion, median (IQR), years & $61.2(57.1-65.1)$ \\
RA disease duration at first RTX, median (IQR), years & $10.1(4.3-29.7)$ \\
ILD disease duration at first RTX, median (IQR), years & $1.9(0.1-4.5)$ \\
Age at RA diagnosis, median (IQR), years & $51.1(40.0-60.6)$ \\
Age at ILD diagnosis, median (IQR), years & $58.3(53.9-63.3)$ \\
Male patient, n (\%) & $16(61.5)$ \\
RF positivity, n (\%) & $25(96.2)$ \\
ACPA positivity, n (\%) & $20(90.9)$ \\
CRP levels, mg/L, mean (SD) & $40.3(58.9)$ \\
ESR levels, mean (SD) & $37.7(18.2)$ \\
Smoking status, n (\%) & $16(64.0)$ \\
Prior TNF inhibitor treatment, n (\%) & $5(19.2)$ \\
DAS28 at first RTX infusion, mean (SD) & $5.3(1.5)$ \\
Radiographic pattern, n (\%) & \\
UIP & $7(26.9)$ \\
NSIP & $8(30.8)$ \\
OP & $2(7.7)$ \\
Indeterminate & $8(30.8)$ \\
Others & $1(3.8)$ \\
Follow-up duration, median (IQR), years & $2.5(1.2-3.7)$
\end{tabular}

RA-ILD = Rheumatoid arthritis-related interstitial lung disease, $R T X=$ rituximab, $R A=$ rheumatoid arthritis, $\mathrm{RF}=$ rheumatoid factor, $\mathrm{ACPA}=$ anti-citrullinated protein antibody, $\mathrm{TNF}=$ tumor necrosis factor, $\mathrm{CRP}=\mathrm{C}$-reactive protein, $\mathrm{ESR}=$ erythrocyte sedimentation rate, $\mathrm{UIP}=$ usual interstitial pneumonia, NSIP = non-specific interstitial pneumonia, $\mathrm{OP}=$ organizing pneumonia

Disclosure of Interests: None declared

DOI: 10.1136/annrheumdis-2021-eular.731

\section{POS0598 COMPARISON OF INJECTION SITE REACTIONS AND INJECTION SITE ERYTHEMA BETWEEN YLB113 AND ETANERCEPT REFERENCE PRODUCT FROM PHASE 3 ACTIVE COMPARATOR STUDY (STUDY NO. YLB113-002)}

D. Stefanidis ${ }^{1}$, U. $G^{2}$, J. Sánchez-Bursón ${ }^{3}$, C. Shah ${ }^{4}$, D. Bakhle ${ }^{4} .{ }^{1}$ Viatris GmbH, Medical Affairs, Bad Homburg, Germany; ${ }^{2}$ Viatris, Biostatistics, Bangalore, India; ${ }^{3}$ Hospital Infanta Luisa, Rheumatology, Sevilla, Spain; ${ }^{4}$ Lupin LTD, Clinical, Pune, India

Background: YLB113 is an etanercept biosimilar approved in all indications of its etanercept Reference Product (RP). Therapeutic equivalence in terms of clinical efficacy, safety and immunogenicity was previously demonstrated in a pivotal multicenter, double-blind, randomized, parallel-group, active-control, comparative study (YLB113-002) in rheumatoid arthritis (RA) subjects. ${ }^{1,2}$ Similar incidence of treatment emergent adverse events (TEAEs) was seen in both treatment arms except for injection site reactions (ISRs) and injection site erythema (ISE), both of which were less frequent in subjects treated with YLB113.

Objectives: This post-hoc analysis was performed to further evaluate the differences in the incidence of ISRs and ISE during the 24 week treatment when the subjects with RA were treated with $50 \mathrm{mg}$ of YLB113 or RP given once a week as a SC injection along with methotrexate.

Methods: Safety analysis set (263 in YLB113 arm and 254 in RP arm) was considered for this analysis.

Local reactions at the site of injection were assessed at each of eight study visits. The number of subjects who experienced ISRs and ISE were statistically compared for YLB113 and RP. The risk difference and 95\% confidence interval $(\mathrm{Cl})$ were computed between arms to understand the magnitude of difference in the incidence of events. The statistical significance of between group difference was tested using chi-square test.

Results: The result of this analysis showed a statistically significant difference in the incidences of ISRs and ISE between subjects who received YLB113 and RP. The risk difference between YLB113 and RP arms for ISR was $-9.98 \%(95 \%$ CI, WALD $-14.81 \%,-5.15 \%)$ and, for ISE it was $-7.94 \%$ (95\% CI, WALD $-11.96 \%$,
- 3.92\%; Table 1). This could be possibly explained by the absence of latex in the syringe needle cap of YLB113.

Table 1. Differences in ISRs and ISEs between YLB113 and Reference Product (RP)

\begin{tabular}{|c|c|c|c|c|}
\hline \multirow[t]{3}{*}{ Event } & \multirow{2}{*}{$\begin{array}{l}\text { YLB113 } \\
\text { N=263 }\end{array}$} & \multirow{2}{*}{$\begin{array}{l}R P \\
N=254\end{array}$} & \multicolumn{2}{|l|}{ YLB113 vs RP } \\
\hline & & & & \\
\hline & $\begin{array}{l}\text { No of subjects } \\
\text { with events [n(\%) }\end{array}$ & $\begin{array}{l}\text { No of subjects } \\
\text { ]ith events } \\
[n(\%)])\end{array}$ & $\begin{array}{l}\text { Risk Diff (95\%Cl, } \\
\text { WALD) }\end{array}$ & $\begin{array}{l}\text { P-value } \\
\text { (Chi- } \\
\text { Square) }\end{array}$ \\
\hline $\begin{array}{l}\text { Injection-site } \\
\text { reactions }\end{array}$ & $10(3.8 \%)$ & $35(13.8 \%)$ & $\begin{array}{l}-9.98 \%(-14.81 \%, \\
-5.15 \%)\end{array}$ & $<0.0001$ \\
\hline $\begin{array}{l}\text { Injection-site } \\
\text { erythema }\end{array}$ & $5(1.9 \%)$ & $25(9.8 \%)$ & $\begin{array}{l}-7.94 \%(-11.96 \% \\
-3.92 \%)\end{array}$ & 0.0001 \\
\hline
\end{tabular}

Conclusion: YLB113 has shown statistically significant lower incidences of ISRs (P-value $<0.0001$ ) and ISEs ( $\mathrm{P}$ value 0.0001$)$ compared to RP. This property may translate to a better acceptability by patients.

\section{REFERENCES:}

[1] EULAR Abstract AB0416 (2019). Ann Rheum Dis, volume 78, supplement 2 , year 2019, page A1670

[2] Yamanaka H, Kamatani N, Tanaka Y, et al. A Comparative Study to Assess the Efficacy, Safety, and Immunogenicity of YLB113 and the Etanercept Reference Product for the Treatment of Patients with Rheumatoid Arthritis. Rheumatol Ther. 2020;7(1):149-163.

[3] Viatris Etanercept Summary of Product Characteritics May 2020

Disclosure of Interests: Dimitris Stefanidis Employee of: Viatris GmbH Sr. Director, Global Medical Affairs Lead, Immunology Biosimilars, Unmesh G Employee of: Viatris, Juan Sánchez-Bursón: None declared, Chirag Shah Shareholder of: As an employee, Shareholder of Lupin LTD, Employee of: Employee of Lupin LTD, Dhananjay Bakhle Shareholder of: As part of Employee Stock Options Plan from Lupin LTD, Employee of: Employee of Lupin LTD

DOI: 10.1136/annrheumdis-2021-eular.829

\section{POS0599 DISEASE ACTIVITY IN PATIENTS WITH RA BY SEROSTATUS AND TREATMENT LINE, FOLLOWING TREATMENT WITH ABATACEPT: RESULTS FROM AN INTERNATIONAL OBSERVATIONAL STUDY}

R. Alten ${ }^{1}$, X. Mariette ${ }^{2}$, R. M. Flipo ${ }^{3}$, R. Caporali ${ }^{4,5}$, M. H. Buch ${ }^{6,7}$, Y. Patel ${ }^{8}$, R. Sanmarti ${ }^{9}$, S. Marsal ${ }^{10}$, M. T. Nurmohamed ${ }^{11}$, H. Griffiths ${ }^{12}$, P. Peichl ${ }^{13}$, B. Bannert ${ }^{14}$, A. Forster ${ }^{15}$, M. Chartier ${ }^{16}$, Y. Elbez $^{17}$, C. Rauch ${ }^{18}$, K. Lozenski ${ }^{19}$, V. Khaychuk ${ }^{20} .{ }^{1}$ Schlosspark-Klinik University, Department of Internal Medicine, Rheumatology, Berlin, Germany; ${ }^{2}$ Université Paris-Saclay, AP-HP, Hospital Bicêtre, Department of Rheumatology, Paris, France; ${ }^{3}$ Centre Hospitalier Universitaire de France, Department of Rheumatology, Lille, France; ${ }^{4}$ University of Milan, Department of Clinical Sciences and Community Health, Milan, Italy; ${ }^{5}$ G. Pini Hospital, Clinical Rheumatology Unit, Milan, Italy;

${ }^{6}$ University of Leeds, Leeds Institute for Rheumatic and Musculoskeletal Medicine, Leeds, United Kingdom; ${ }^{7}$ University of Manchester, Division of Musculoskeletal \& Dermatological Sciences, Manchester, United Kingdom; ${ }^{8}$ Hull Royal Infirmary, Department of Rheumatology, Hull, United Kingdom ${ }^{9}$ Hospital Clínic de Barcelona, Rheumatology Department, Barcelona, Spain; ${ }^{10}$ Hospital Universitari Vall d'Hebron, Rheumatology, Barcelona, Spain; ${ }^{11} A R C$ Amsterdam University Hospitals - VU University Medical \& Reade, Department of Rheumatology, Amsterdam, Netherlands; ${ }^{12}$ University Hospital Geelong, Barwon Rheumatology Service, Geelong, Australia; ${ }^{13}$ Evangelical Hospital, Department of Internal Medicine, Vienna, Austria; ${ }^{14}$ Universitätsspital Basel, Rheumatologische Universitätsklinik, Basel, Switzerland; ${ }^{15}$ Schulthess Klinik, Department of Rheumatology, Zürich, Switzerland; ${ }^{16}$ Bristol Myers Squibb, Non-Registrational Data Generation, Rueil-Malmaison, France; ${ }^{17}$ Deepscover, Biostatistics, Puteaux, France ${ }^{18}$ Bristol Myers Squibb, Medical Immunology \& Fibrosis, Munich, Germany; ${ }^{19}$ Bristol Myers Squibb, Immunology and Fibrosis, Princeton, United States of America; ${ }^{20}$ Bristol Myers Squibb, US Medical Immunology and Fibrosis, Princeton, United States of America

Background: RF and anti-citrullinated protein antibodies (ACPAs) are associated with a severe and aggressive disease course in patients with RA. ${ }^{1}$ Abatacept is a selective co-stimulation modulator for the treatment of RA. ${ }^{2}$ ASCORE (Abatacept SubCutaneOus in Routine Clinical PracticE; NCT02090556) was a 2-year, observational, prospective, multicentre study of SC abatacept for the treatment of RA in routine clinical practice. ${ }^{3}$

Objectives: To determine if serostatus and treatment line impacted disease activity in patients enrolled in the ASCORE study. 